\title{
Electronic transport properties of double-wall carbon nanotubes
}

\author{
Subhadeep Datta, ${ }^{1}$ Shidong Wang, ${ }^{2}$ Carmen Tilmaciu, ${ }^{3}$ Emmanuel Flahaut, ${ }^{4}$ Laëtitia Marty, ${ }^{1}$ Milena Grifoni, ${ }^{5}$ and \\ Wolfgang Wernsdorfer ${ }^{1}$ \\ ${ }^{1}$ Institut Néel, CNRS and Université Joseph Fourier, BP 166, F-38042 Grenoble Cedex 9, France \\ ${ }^{2}$ Department of Mechanical Engineering and Materials Science, Duke University, Durham, North Carolina 27708, USA \\ ${ }^{3}$ Université de Toulouse, UPS, INP, Institut Carnot Cirimat, 118 route de Narbonne, F-31062 Toulouse Cedex 9, France \\ ${ }^{4}$ CNRS, Institut Carnot Cirimat, F-31062 Toulouse, France \\ ${ }^{5}$ Theoretische Physik, Universität Regensburg, D-93040 Regensburg, Germany
}

(Received 10 April 2011; published 20 July 2011)

\begin{abstract}
We studied the discretized electronic spectra of double-wall carbon nanotube (DWCNT) quantum dots (QDs) in the Coulomb-blockade regime. At low temperatures, the stability diagrams show a clear and regular eight-electron periodicity, which is due to the nonzero intershell couplings. Furthermore, the electronic charging energy, the energy level spacing, and the intershell coupling strengths of the measured DWCNT QDs were determined.
\end{abstract}

DOI: 10.1103/PhysRevB.84.035408

PACS number(s): 73.22.-f, 61.48.De, 73.63.Fg, 85.35.Kt

\section{INTRODUCTION}

Electronic transport properties in nanostructures, such as carbon nanotube (CNT) quantum dots (QDs), ${ }^{1-3}$ single molecules, ${ }^{4}$ semiconductor nanocrystals,${ }^{5}$ metallic nanoparticles, ${ }^{6}$ and graphene ribbons ${ }^{7}$ are strongly dominated by the single-electron charging and energy-level quantization. Electronic spectroscopy at low temperature provides detailed information on their quantum-level structures. Because of their finite size, QDs exhibit many properties similar to natural atoms. ${ }^{8}$ For example, quantum confinement induces discretized electronic energy spectra with shell structures, and electrons can be shared between coupled QDs similar to molecular bondings. One of the advantages with respect to natural atoms is that the confinement potential can be electrically tuned by a gate electrode. ${ }^{8}$

Single-wall carbon nanotubes (SWCNTs), which can be viewed as a graphene sheet wrapped into a cylinder, ${ }^{9}$ are convenient systems to study these features due to their simple geometries with less spatial imperfection and weak spin-orbit coupling compared to other QD systems. ${ }^{2,10,11}$ In the Coulombblockade regime, devices based on SWCNT QDs connected to metallic leads show regular Coulomb-blockade patterns, which originate from their electronic structures. ${ }^{2}$ Because of the spin degeneracy of two bands near the Fermi points, the stability diagrams of SWCNT QDs exhibit four-electron periodicity of the Coulomb diamond patterns. ${ }^{12,13}$

Among many possible applications, SWCNTs can be used to study the spin reversal of individual molecular quantum magnets. ${ }^{14}$ Supramolecular spin-valve devices of SWCNT exhibited magnetoresistance ratios up to $300 \%$ between fully polarized and nonpolarized molecules. ${ }^{15}$ SWCNTs can also be filled with magnetic nanoparticles ${ }^{16}$ establishing new magneto-Coulomb effects. ${ }^{17}$

Besides SWCNTs, multi-wall carbon nanotubes (MWCNTs) consist of two or more concentric walls. Although transport properties of SWCNT QDs in the Coulomb-blockade regime have been well understood, the energy spectra of MWCNT QDs still lack full understanding. ${ }^{18}$ The presence of multiple shells complicates the transport properties because (1) it increases the number of conducting channels, (2) it induces the possibility of interactions between the different walls, and (3) it makes the influence of defects more important as MWCNTs become more susceptible to them.

Double-wall carbon nanotubes (DWCNTs) with two concentric walls are the simplest MWCNTs and therefore the best candidates to study their electrical transport properties. ${ }^{19}$ Depending on the ratio between the unit cell lengths of the two shells, a DWCNT may be either commensurate (c-DWCNT) or incommensurate (i-DWCNT). At low energy, nonzero intershell coupling occurs in c-DWCNTs while intershell coupling vanishes in i-DWCNTs. ${ }^{20-24}$ As explained theoretically in Ref. 25, nonzero intershell coupling causes eightelectron periodicity in the stability diagram of c-DWCNT while four-electron periodicity, similar to that in SWCNT QDs, is observed in case of zero intershell coupling in i-DWCNT. Mixing of orbital states of the two walls is the main source of nonzero intershell coupling in DWCNTs in spite of the effects from disorder present in the system. ${ }^{10,26,27}$ In Ref. 28, an eight-electron periodicity was indeed observed in DWCNT QDs. However, the coexistence of both four- and eight-electron periodicities is puzzling and requires further studies.

In this work, we report on a systematic electron transport study of DWCNT QDs. We focus more specifically on the devices with stability diagrams showing only eight-electron periodicity, which is specific to DWNTs and means nonzero intershell coupling in our DWCNT QDs. By using the model of Ref. 25, we determine the possible schemes for single-electron tunneling processes. Furthermore, we extract all the important parameters of DWCNT QDs, namely, electron-addition energy $E_{\text {add }}$, energy level spacing $\epsilon_{0}$, and the intershell coupling strengths. To our knowledge, the values of the intershell coupling strengths were not extracted before from electrontransport-spectroscopy measurements. By using the excitation lines, we also determine the full energy shell and partially filled QD states.

In Sec. II A, we present experimental details of sample preparation and measurements while we describe our theoretical model in Sec. II B. Results and discussions are given in Sec. III and conclusions are drawn in Sec. IV. 


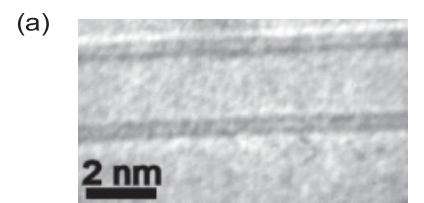

(b)
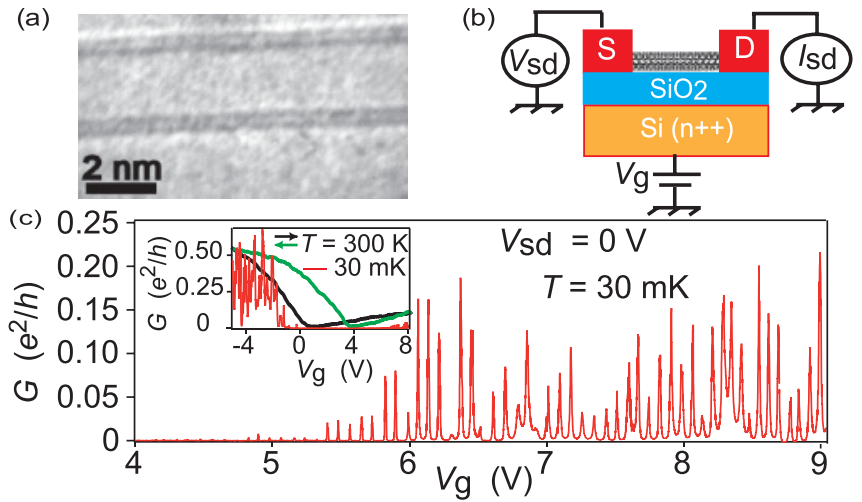

FIG. 1. (Color online) (a) HRTEM picture (JEOL-JEM-2100) of a double-wall carbon nanotube (DWCNT). (b) Schematic diagram of the device with a DWCNT quantum dot (QD) contacted to Pd electrodes. The $\mathrm{Si}$ substrate with a $400-\mathrm{nm} \mathrm{SiO}_{2}$ layer acts as a gate electrode. (c) Linear-response differential conductance, $G$, vs the gate voltage, $V_{g}$, at $30 \mathrm{mK}$ of a $400 \mathrm{~nm}$ long DWCNT QD. Conductance oscillations due to Coulomb blockade can be observed. Inset: Ambipolar behavior of transfer characteristics at $300 \mathrm{~K}$ and Fabry-Perot conductance oscillations of hole transport at $30 \mathrm{mK}$.

\section{EXPERIMENTAL METHODS AND THEORETICAL MODEL}

\section{A. Sample preparation and measurement}

Isolated DWCNTs were synthesized by catalytic-chemicalvapor-deposition (CCVD) method using $\mathrm{Mg}_{0.9} \mathrm{Co}_{0.1} \mathrm{O}$ solid solutions as a starting material. ${ }^{29}$ The distribution of the occurrence of each number of walls from high-resolution transmission electron microscopy (HRTEM) shows that $80 \%$ of the CNTs are double walled as shown in Fig. 1(a). The median inner diameter is about $1.35 \mathrm{~nm}$ and the outer one is about $2.05 \mathrm{~nm}$. CNTs are then dispersed on a highly doped silicon substrate capped by a 400-nm silicon-dioxide layer, which acts as the gate electrode to modulate the charge density within the CNTs [c.f. Fig. 1(b)]. CNT junctions with typical length of about $300 \mathrm{~nm}$ are fabricated using electron beam lithography. CNTs are connected to 50-nm-thick Pd electrodes, which act as source-drain leads. Only those circuits of room-temperature resistances between 50 to $500 \mathrm{k} \Omega$ are used in the experiments. Devices are cooled down in a ${ }^{3} \mathrm{He} /{ }^{4} \mathrm{He}$ dilution refrigerator with a base temperature of $30 \mathrm{mK}$. Conventional two-probe measurements are performed by recording the differential conductance, $G=d I / d V$, when changing both the gate, $V_{g}$, and the source-drain, $V_{\text {sd }}$, voltages.

At room temperature, the transfer characteristics show ambipolar behavior with "on-off" ratio of about 20, as shown in the inset of Fig. 1(c). The different transparency of the $n$ - and $p$-type regions is visible on the inset of Fig. 1(c) from Ref. 3. The appearance of quasiperiodic conductance oscillations at $30 \mathrm{mK}$ in Fig. 1(c) features Coulomb blockade. Presence of a large gap with zero conductance indicates the existence of semiconducting shells. This in fact reduces the influence of disorder on intershell coupling between two walls in our device. ${ }^{27}$ Measurements of conductance while sweeping the bias voltage (from -10 to $10 \mathrm{mV}$ ) for a set of different values of gate voltages (from 5.2 to $8.0 \mathrm{~V}$ ) are shown in Fig. 2(a). Coulomb blockade diamonds and excited states can be observed on a set of more than 30 diamonds in this range. A closer inspection shows that the size of the Coulomb diamonds varies as the electron number increases. In Fig. 2(b), we can clearly identify eight-electron periodicity in the diamond patterns. The numbers inside the diamonds indicate the additional electron filling the DWCNT QD. Addition energy $\left(E_{\text {add }}\right)$ is extracted from the heights of the Coulomb diamonds and shown as a function of an additional electron number in Fig. 2(c). Eight-electron periodicity is clearly observed in three consecutive sets of diamond. In each set, there is a distribution of addition energies, which will be explained later on by considering interwall couplings. Addition energies for the even-numbered electrons (2nd, 4th, 6th, and 8th) in every set are almost the same because only the charging energy is needed to put a second electron on the same orbital state, similar to SWCNTs. ${ }^{30}$ Similar sets of Coulomb diamonds revealing only eight-fold symmetry have been observed for 10 devices among the more than 20 measured.

From the stability diagram in Fig. 2(b), we can estimate some of the relevant parameters of the QD: charging energy $U_{c}=14 \mathrm{meV}$ and level spacing $\epsilon_{0}=8 \mathrm{meV}$. Calculated from the geometry of the device, the charging energy, $U_{c}$, is about $13.5 \mathrm{meV}$, which is in accordance with the previous value deduced from the diamond height. The capacitive coupling between the DWCNT QD and the electrodes is related to the

(a)

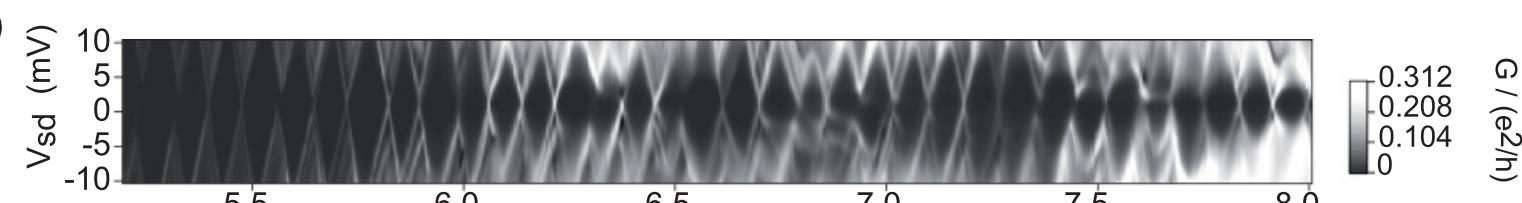

(b)

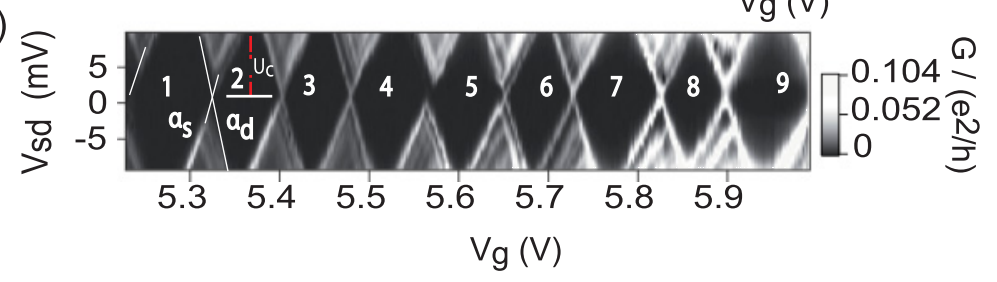

(c)

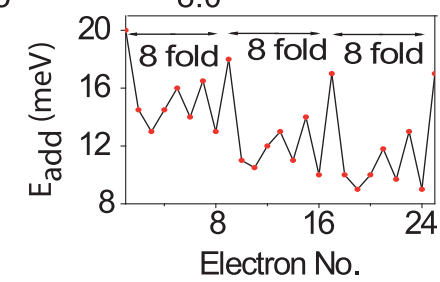

FIG. 2. (Color online) (a) Stability diagram of a double-wall carbon nanotube (DWCNT) quantum dot (QD). An eight-electron periodicity can be clearly observed. (b) Zoom-in image of one set of Coulomb diamonds. (c) Addition energy, $E_{\text {add }}$, vs the extra number of electrons in the QD. 
slopes of the lines defining a diamond [c.f. Fig. 2(b)]. They are estimated to be about $\alpha_{s}=0.25$ and $\alpha_{d}=-0.42$. Therefore, the source capacitance is $C_{s}=4.40 \mathrm{aF}$, the drain capacitance $C_{d}=5.56 \mathrm{aF}$, and the gate capacitance $C_{g}=1.84 \mathrm{aF}$ by using the relations ${ }^{8} \alpha_{s}=C_{g} /\left(C-C_{s}\right)$ and $\alpha_{d}=-C_{g} / C_{s}$, where the total capacitance $C=\left(C_{s}+C_{d}+C_{g}\right)=11.80 \mathrm{aF}$. The slopes, $\alpha_{s, d, g}$, correspond also to aligning the electrochemical potentials of the QD with the source (source, drain, gate) voltages, respectively. We find that $\alpha_{g}=C_{g} / C=0.15$. The period of the conductance peak distances is then estimated to be $\Delta V_{g}=U_{c} /\left(e \alpha_{g}\right)=0.10 \mathrm{~V}$, comparable to the observed value $\Delta V_{g}=0.09 \mathrm{~V}$ [c.f. Fig. 2(b)].

\section{B. Theoretical Model}

We model the DWCNT QD systems by using the method in Ref. 25. Here, we only give a brief description and more details of the method can be found in the reference. The properties of DWCNT QDs can be investigated by using the Bosonization method, including the forward-scattering exactly. ${ }^{23,31,32}$ The Hamiltonian of a DWCNT QD can then be separated into two parts, $H=H_{f}+H_{b}$. One part, $H_{f}$, describes the ground state and Fermionic excitations while the other part, $H_{b}$, the bosonic excitations. The Fermionic Hamiltonian is similar to the constant-interaction model as

$$
\begin{aligned}
H_{f}= & \sum_{\alpha r \sigma} \operatorname{sgn}(r) \Delta \epsilon_{0} \mathcal{N}_{\alpha r \sigma}+\left(\alpha \zeta \epsilon_{0}-\frac{1}{2} \epsilon_{0}\right) \mathcal{N}_{\alpha r \sigma} \\
& +\frac{1}{2} \epsilon_{0} \mathcal{N}_{\alpha r \sigma}^{2}+\frac{1}{2} \sum_{\alpha \alpha^{\prime}} U_{c}\left(\sum_{r \sigma} \mathcal{N}_{\alpha r \sigma}\right)\left(\sum_{r^{\prime} \sigma^{\prime}} \mathcal{N}_{\alpha^{\prime} r^{\prime} \sigma^{\prime}}\right),
\end{aligned}
$$

where $\operatorname{sgn}()$ is the sign function, $\alpha= \pm$ denotes the bonding (antibonding) states, $r=R / L= \pm$ is the index of right- and left-moving electrons, and $\sigma= \pm$ is the spin index. The number operator $\mathcal{N}_{\alpha r \sigma}$ gives the number of electrons in state $(\alpha r \sigma)$. There are four orbital Fermionic modes with spin degeneracy, which we label as $L_{ \pm}$and $R_{ \pm}$[c.f. Fig 3(a)]. Their energy spectra are characterized by the energy level spacing, $\epsilon_{0}$, the charging energy, $U_{c}$, the intershell coupling, $\zeta \epsilon_{0}$, with a dimensionless parameter $0 \leqslant \zeta<0.5$, and a dimensionless Fermi-point-mismatching parameter $0 \leqslant \Delta<0.5$.

The Bosonic Hamiltonian can also be diagonalized as

$$
H_{b}=\sum_{q>0} \sum_{j \delta \xi} \epsilon_{j \delta \xi}(q) a_{j \delta \xi q}^{\dagger} a_{j \delta \xi q},
$$

where $a^{\dagger}(a)$ is the bosonic creation (annihilation) operator, $q$ is a quantum number, $j=c, s$ is the index of charge (spin) modes, and $\delta, \xi= \pm$ define total (relative) modes with respect to the branch degree of freedom. Because of the one-dimensional characteristics of DWCNT energy spectrum and Coulomb interactions, there are eight Bosonic excitations modes besides the Fermionic ones. Out of these eight Bosonic modes, only the energy dispersions of the two highest excitation modes depend on the Coulomb interactions. The energy dispersions of the six "neutral" modes are the same as for the noninteracting Fermionic system. In the simulations, only Fermionic states and these six neutral Bosonic excitation modes are included as we consider only lowest excitations.

After obtaining the energy spectrum of a DWCNT QD, its electronic transport properties are calculated by using the

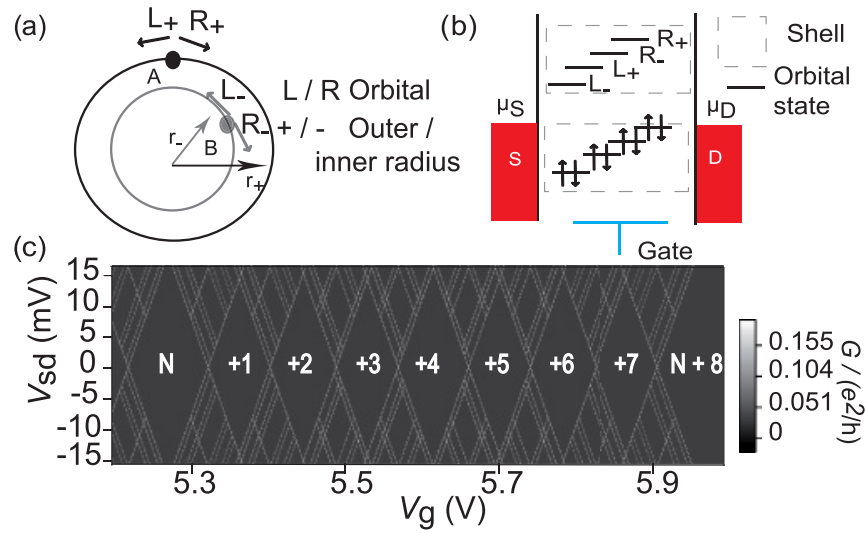

FIG. 3. (Color online) (a) Schematic diagram of the cross section of a DWCNT. Atoms $\mathrm{A}$ and $\mathrm{B}$ in two shells of radii $R_{+}$and $R_{-}$, respectively, are projected onto this cross section. There are two Fermi points and two branches $L / R$ with left-moving right-moving \pm electrons at each Fermi point. (b) Schematic energy spectrum of a double-wall carbon nanotube (DWCNT) quantum dot (QD) with nonzero intershell interactions. Energy levels are in the order: $L_{-}$, $L_{+}, R_{-}$, and $R_{+}$, corresponding to our measurements. (c) Simulated stability diagrams of a DWCNT QD, showing an eight-electron periodicity. Parameters used are: $U_{c}=14 \mathrm{meV}, \epsilon_{0}=8 \mathrm{meV}, \Delta=$ 0.06 , and $\zeta=0.18$. The intershell coupling strength is $1.44 \mathrm{meV}$.

Liouville equation for the reduced-density-matrix approach to the lowest order in the tunneling rate. This approximation is justified as the measured DWCNT QDs are in the Coulombblockade regime. Furthermore, we assume that only inelastic tunneling processes occur in the DWCNT QD and the outer shell is connected to both leads with same tunneling rates. Although only DWCNT QDs with two metallic shells are considered in Ref. 25, the method proposed there is also valid for DWCNT QDs with semiconducting shells provided that the electrochemical potentials are away from the band gap, which is the case in our experiments [c.f. Fig. 2(a)].

\section{RESULTS AND DISCUSSIONS}

The observed eight-electron periodicity of addition energies in Fig. 2(c) can be understood by obtaining the addition energy from the Hamiltonian of the DWCNT QD. From the Fermionic Hamiltonian of DWCNT QD, Eq. (1), the addition energy, which is defined as $E_{\text {add, } N}=\left|\mu_{N}-\mu_{N-1}\right|$, has an eight-electron periodicity, ${ }^{25}$

$$
\begin{gathered}
E_{\text {add }, 1}=E_{\text {add }, 3}=E_{\text {add }, 5}=E_{\text {add }, 7}=U_{c}, \\
E_{\text {add }, 2}=E_{\text {add }, 6}=2 \min (\Delta, \zeta) \epsilon_{0}+U_{c}, \\
E_{\text {add }, 4}=2|\Delta-\zeta| \epsilon_{0}+U_{c}, \\
E_{\text {add }, 8}=\epsilon_{0}-2(\Delta+\zeta) \epsilon_{0}+U_{c} .
\end{gathered}
$$

From the experimental addition energies shown in Fig. 2(c), we find the following set of parameters: the charging energy $U_{c}=14.0 \mathrm{meV}$, the energy level spacing $\epsilon_{0}=8.0 \mathrm{meV}$, the mismatch parameter $\Delta=0.06$, and the intershell coupling parameter $\zeta=0.18$, that is, an intershell coupling of $1.44 \mathrm{meV}$. The coupling strength between DWCNT QD and source and drain leads is $2.1 \times 10^{-3} \mathrm{meV}$. By fitting to the value of measured conductances, the tunneling rate $\gamma$ is 

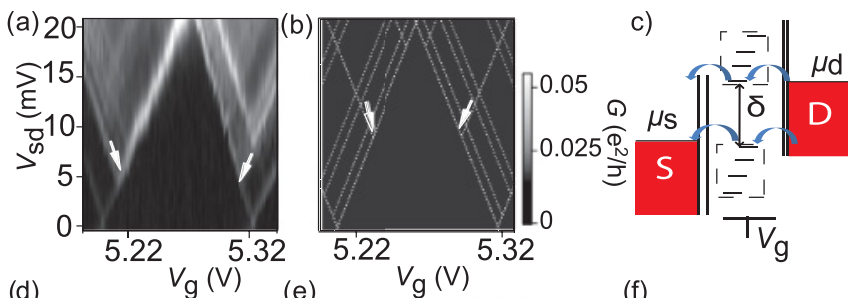

(d)
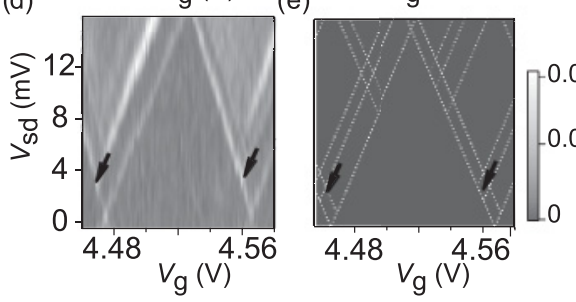

(f)

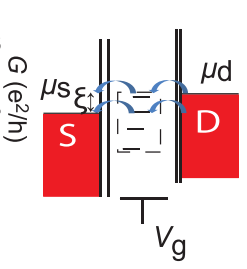

FIG. 4. (Color online) Experimental (a) and (d) and simulated (b) and (e) excitation spectra for different electron numbers at $30 \mathrm{mK}$ in a double-wall carbon nanotube (DWCNT) quantum dot (QD). (c) and (f) Schematic diagrams of tunneling processes between states in a same energy shell (f) and in different ones (c), which cause two different sets of excitation lines visible in (a) and (d). In (a), the symmetry of the excitation lines intersecting the two diamond edges at the position pointed by arrows indicate a QD with full energy shell at this range of gate voltage. The nonsymmetric features observed in (d) indicate a partially filled QD.

estimated to be about $3 \times 10^{9} \mathrm{~s}^{-1}$, well comparable with the experimental value of $1 \times 10^{9} \mathrm{~s}^{-1}$ [extracted from data shown in Fig. 1(c)]. Using these parameters, the simulated stability phase diagram is shown in Fig. 3(c). It has an eight-fold periodicity and the shapes of the diamonds agree well with the experimental ones shown in Fig. 2(b). By substituting the values of fit parameters into the Hamiltonian Eq. (1), we find that the filling sequence in the measured DWCNT QDs is $L_{-} \rightarrow L_{+} \rightarrow R_{-} \rightarrow R_{+}$, as shown in Fig. 3(b).

The energy spectra in DWCNT QDs have shell-like structure, ${ }^{33}$ and it is useful to distinguish the QD with full or partially filled energy shell. We now compare the excitation spectra (see Fig. 4) for two different Coulomb diamonds, the larger one and one inside the same shell. The first and second columns in Fig. 4 show the Coulomb diamonds from experiment and simulation, respectively, while the last one depicts the scheme of the single-electron tunneling process through an excited state. Lines terminating on diamond sides involve excited states of this charge state. Their pattern provides a complete spectroscopy of the energy levels, and the vertical distance between the lines is a direct measurement of the level spacing. ${ }^{8}$ The diamond of Figs. 4(a) and 4(b) exhibits symmetric excitation features on the two sides, which occurs either for a full or a half-filled shell [see Fig. 3(c)]. If we now consider a different diamond [see Figs. 4(d) and 4(e)], such symmetry is broken corresponding to a partially filled shell $(N \neq 0$ or 4 [8]). The excited-states pattern corresponds to the expected one for a +5 electron charge state [see Fig. 3(c)]. Moreover, the white arrows in Figs. 4(a) and 4(b) indicate the excited-state level spacing $(\sim 5 \mathrm{meV})$, which is much larger than the level spacing we observe in case of Figs. 4(d) and 4(e) $(\sim 3 \mathrm{meV})$. As the level spacing is larger between two shells $(\delta)$ as compared to within a same shell ( $\xi)$, this confirms that the case in Figs. 4(a) and 4(b) corresponds to a full-shell charge state.

\section{CONCLUSIONS}

In conclusion, we have observed eight-electron periodicity in the single-particle stability diagram of DWCNT QDs. The charging energy, level spacing, intershell coupling strength, mismatching parameters, and coupling strength to the leads are obtained. The full energy shell and partially filled shell states are determined by examining the excitation lines. The extraction of the intershell coupling strengths in DWCNTs from the spectroscopy measurements was never performed before. The simple and standard spectroscopy measurement provides a relatively easy approach to investigate and to better understand the intershell couplings in DWCNTs and MWCNTs, which are crucial for their application in nanodevices.

\section{ACKNOWLEDGMENTS}

This work is partially supported by the ANR-PNANO project MolNanoSpin No. ANR-08-NANO-002, ERC Advanced Grant MolNanoSpin No. 226558, STEP MolSpinQIP, and especially S.D. acknowledges the financial support from the Nanosciences Foundation of Grenoble. Samples were fabricated in the NANOFAB facility of the Néel Institute. We thank F. Balestro, N. Bendiab, L. Bogani, E. Bonet, T. Crozes, E. Eyraud, R. Haettel, C. Hoarau, D. Lepoittevin, V. Reita, C. Thirion, and especially R. Maurand.
${ }^{1}$ S. J. Tans, M. H. Devoret, H. Dai, A. Thess, R. E. Smalley, L. J. Geerligs, and C. Deeker, Nature (London) 386, 474 (1997).

${ }^{2}$ W. Liang, M. Bockrath, and H. Park, Phys. Rev. Lett. 88, 126801 (2002).

${ }^{3}$ J. Cao, Q. Wang, and H. Dai, Nat. Mater. 4, 745 (2005).

${ }^{4}$ H. Park, J. Park, A. Lim, E. Anderson, A. Alivisatos, and P. McEuen, Nature (London) 407, 57 (2000).

${ }^{5}$ D. Klein, R. Roth, A. Lim, A. Alivisatos, and P. McEuen, Nature (London) 389, 699 (1997).

${ }^{6}$ D. C. Ralph, C. T. Black, and M. Tinkham, Phys. Rev. Lett. 78, 4087 (1997).
${ }^{7}$ A. Candini, S. Klyatskaya, M. Ruben, W. Wernsdorfer, and M. Affronte, Nano Lett. 11, 2634 (2011).

${ }^{8}$ R. Hanson, L. P. Kouwenhoven, J. R. Petta, S. Tarucha, and L. M. K. Vandersypen, Rev. Mod. Phys. 79, 1217 (2007).

${ }^{9}$ J.-C. Charlier, X. Blase, and S. Roche, Rev. Mod. Phys. 79, 677 (2007).

${ }^{10}$ F. Triozon, S. Roche, A. Rubio, and D. Mayou, Phys. Rev. B 69, 121410 (2004).

${ }^{11}$ D. H. Cobden and J. Nygård, Phys. Rev. Lett. 89, 046803 (2002).

${ }^{12}$ E. Minot, Y. Yaish, V. Sazonova, and P. McEuen, Nature (London) 428, 536 (2004). 
${ }^{13}$ S. Zaric, G. Ostojic, J. Kono, J. Shaver, V. Moore, M. Strano, R. Hauge, R. Smalley, and X. Wei, Science 304, 1129 (2004).

${ }^{14}$ L. Bogani and W. Wernsdorfer, Nat. Mater. 7, 179 (2008).

${ }^{15}$ M. Urdampilleta, S. Klyatskaya, J.-P. Cleuziou, M. Ruben, and W. Wernsdorfer, Nat. Mater. 10, 502 (2011).

${ }^{16}$ J. P. Cleuziou, W. Wernsdorfer, T. Ondarcuhu, and M. Monthioux, ACS NANO 5, 2348 (2011).

${ }^{17}$ S. Datta, L. Marty, J. P. Cleuziou, C. Tilmaciu, B. Soula, E. Flahaut, and W. Wernsdorfer (submitted).

${ }^{18}$ M. R. Buitelaar, A. Bachtold, T. Nussbaumer, M. Iqbal, and C. Schönenberger, Phys. Rev. Lett. 88, 156801 (2002).

${ }^{19}$ S. Wang, X. Liang, Q. Chen, Z. Zhang, and L.-M. Peng, J. Phys. Chem. B 109, 17361 (2005).

${ }^{20}$ R. Saito, G. Dresselhaus, and M. Dresselhaus, J. Appl. Phys. 73, 494 (1993).

${ }^{21}$ S. Roche, F. Triozon, A. Rubio, and D. Mayou, Phys. Rev. B 64, 121401 (2001).

${ }^{22}$ S. Uryu, Phys. Rev. B 69, 075402 (2004).
${ }^{23}$ S. Wang and M. Grifoni, Phys. Rev. Lett. 95, 266802 (2005).

${ }^{24}$ S. Roche, F. Triozon, R. Angel, and D. Mayou, Phys. Lett. A 285, 94 (2001).

${ }^{25}$ S. Wang and M. Grifoni, Phys. Rev. B 77, 085431 (2008).

${ }^{26}$ P. Poncharal, C. Berger, Y. Yi, Z. L. Wang, and W. A. de Heer, J. Phys. Chem. B 106, 12104 (2002).

${ }^{27}$ S. Roche, F. Triozon, and R. Angel, Appl. Phys. Lett. 79, 3690 (2001).

${ }^{28}$ S. Moon, W. Song, J. S. Lee, N. Kim, J. Kim, S.-G. Lee, and M.-S. Choi, Phys. Rev. Lett. 99, 176804 (2007).

${ }^{29}$ E. Flahaut, R. Bacsa, A. Peigney, and C. Laurent, Chem. Commun. 2003, 1442.

${ }^{30}$ S. Sapmaz, P. Jarillo-Herrero, J. Kong, C. Dekker, L. P. Kouwenhoven, and H. S. J. van der Zant, Phys. Rev. B 71, 153402 (2005).

${ }^{31}$ R. Egger and A. O. Gogolin, Phys. Rev. Lett. 79, 5082 (1997).

${ }^{32}$ L. Mayrhofer and M. Grifoni, Phys. Rev. B 74, 121403 (2006).

${ }^{33}$ S. M. Reimann and M. Manninen, Rev. Mod. Phys. 74, 1283 (2002). 